\section{Baseline Vitamin D Status, Sleep Patterns, and the Risk of Incident Type 2 Diabetes in Data From the UK Biobank Study}

Diabetes Care 2020;43:2776-2784 | https://doi.org/10.2337/dc20-1109

\section{OBJECTIVE}

Circulating vitamin $D$ concentrations have been associated with the risk of type 2 diabetes (T2D), but the results are inconsistent. Emerging evidence suggests that vitamin D metabolism is linked to sleep behaviors. We investigated the prospective association between serum 25-hydroxyvitamin D (25OHD) and the risk of incident T2D and whether such association was modified by sleep behaviors.

\section{RESEARCH DESIGN AND METHODS}

The study included 350,211 individuals free of diabetes in the UK Biobank. Serum 250HD (nmol/L) concentrations were measured. Five sleep behaviors including sleep duration, insomnia, snoring, chronotype, and daytime sleepiness were included to generate overall sleep patterns, defined by healthy sleep scores. We also calculated genetic risk scores of sleep patterns.

\section{RESULTS}

During a median follow-up of 8.1 years, we documented 6,940 case subjects with incident T2D. We found that serum 250HD was significantly associated with a lower risk of incident T2D, and the multivariate adjusted hazard ratio (HR) (95\% $\mathrm{Cl}$ ) per $10 \mathrm{nmol} / \mathrm{L}$ increase was $0.88(0.87-0.90)$. We found a significant interaction between 250HD and overall sleep patterns on the risk of incident T2D ( $P$ for interaction $=$ 0.002). The inverse association between high 25OHD and T2D was more prominent among participants with healthier sleep patterns. Among the individual sleep behaviors, daytime sleepiness showed the strongest interaction with 250HD ( $P$ for interaction $=0.0006$ ). The reduced $\mathrm{HR}$ of T2D associated with high 250HD appeared to be more evident among participants with no frequent daytime sleepiness compared with those with excessive daytime sleepiness. The genetic variations of the sleep patterns did not modify the relation between 25OHD and T2D.

\section{CONCLUSIONS}

Our study indicates that higher serum 250HD concentrations are associated with a lower risk of incident T2D, and such relations are modified by overall sleep patterns, with daytime sleepiness being the major contributor.

Vitamin $D$ is a fat-soluble steroid hormone essential for calcium homeostasis and bone health $(1,2)$. Several randomized controlled trials have explored the role of supplemental vitamin $\mathrm{D}$ in preventing cancer and cardiometabolic diseases in recent years (3-5). However, the effects of vitamin D supplementation on the risk of type 2 diabetes
Mengying Wang, ${ }^{1,2}$ Tao Zhou, ${ }^{2}$ Xiang $\mathrm{Li}^{2}$ Hao Ma, ${ }^{2}$ Zhaoxia Liang, ${ }^{2}$

Vivian A. Fonseca, ${ }^{3}$ Yoriko Heianza, ${ }^{2}$ and Lu $Q i^{2,4,5}$
${ }^{1}$ Department of Epidemiology and Biostatistics, School of Public Health, Peking University, Beijing, China

${ }^{2}$ Department of Epidemiology, School of Public Health and Tropical Medicine, Tulane University, New Orleans, $L A$

${ }^{3}$ Section of Endocrinology, Department of Medicine, School of Medicine, Tulane University, New Orleans, $L A$

${ }^{4}$ Department of Nutrition, Harvard T.H. Chan School of Public Health, Boston, MA

${ }^{5}$ Channing Division of Network Medicine, Department of Medicine, Brigham and Women's Hospital and Harvard Medical School, Boston, MA

Corresponding author: Lu Qi, 1qil@tulane.edu

Received 11 May 2020 and accepted 24 July 2020

This article contains supplementary material online at https://doi.org/10.2337/figshare.12746681.

(C) 2020 by the American Diabetes Association. Readers may use this article as long as the work is properly cited, the use is educational and not for profit, and the work is not altered. More information is available at https://www. diabetesjournals .org/content/license. 
(T2D) were mixed across intervention studies (4-6). For example, the Vitamin D and Type 2 Diabetes (D2d) randomized controlled trial showed that vitamin $D_{3}$ supplementation did not lower the risk of T2D among persons with prediabetes (4). In addition, a body of previous prospective studies have associated serum levels of 25hydroxyvitamin D (25OHD), the best measure of vitamin D status, with T2D (7-10). Several meta-analyses of prospective cohort studies showed a reduced risk of T2D associated with high circulating vitamin D levels, yet statistically significant heterogeneity in these observations was detected (11-13). The inconsistent associations might be partly due to the modification effects of varying lifestyle factors related to vitamin $\mathrm{D}$ status across the studies.

Emerging evidence has linked various sleep behaviors with vitamin D metabolism (14-16). For example, short sleep duration and obstructive sleep apnea were associated with 250HD deficiency $(16,17)$. Subjects with excessive daytime sleepiness were found to have an increased likelihood of low serum $250 \mathrm{HD}$ concentrations (15), possibly due to inadequate outdoor sunlight exposure that reduces the biosynthesis of vitamin $D$ (18). Notably, unhealthy sleep behaviors have also been related to an increased risk of T2D $(19,20)$. Therefore, we hypothesized that sleep behaviors might modify the association between $250 \mathrm{HD}$ and the risk of T2D.

In this prospective study of 350,211 participants from the UK Biobank, we tested the association between serum 25OHD and incident T2D and particularly examined the potential modification effect of our recently developed overall sleep patterns, which incorporated sleep duration, chronotype, insomnia, snoring, and excessive daytime sleepiness (21). We also assessed the modification effect of the genetically determined sleep behaviors.

\section{RESEARCH DESIGN AND METHODS}

Study Design and Population

The detailed study design and methods of the UK Biobank study have been described elsewhere (22). In brief, UK Biobank recruited $>500,000$ community-based volunteers aged 40-69 years across the U.K. between 2006 and 2010. Participants provided health information through touchscreen questionnaires and anthropometric measurements. Blood samples were collected for genotyping and biomarker analyses. All participants gave written consent, and ethical approval was obtained from the North West Multi-Centre Research Ethics Committee (London, U.K.).

In the current study, we excluded those with diabetes at baseline $(n=27,258)$ and those with missing values on serum 25OHD concentrations ( $n=51,634$ ) or sleep variables ( $n=74,303$ ) at baseline, leaving a total of 350,211 participants in the main analysis. In the genetic analysis, we used the UK Biobank genetic data of 274,356 unrelated individuals of European descent.

\section{Assessment of Serum 250HD Concentrations}

A range of biochemistry markers was measured in the blood sample collected at baseline (2006-2010). Serum vitamin $D$ (nanomoles per liter) was measured by chemiluminescence technology analysis on a DiaSorin Ltd. LIAISON XL. Calibration and quality control were conducted by the UK Biobank. In brief, coefficients of variation for low, medium, and high internal quality control level samples for each biomarker were provided. The coefficients of variation for serum $250 \mathrm{HD}$ ranged from 5.0 to $6.1 \%$. In addition, the external quality assurance result was $100 \%$ for vitamin D. The detailed information of the measurements is provided on the UK Biobank website (https://biobank .ndph.ox.ac.uk/showcase/showcase/docs/ serum_biochemistry.pdf).

\section{Assessment of Sleep Behaviors}

The information on sleep behaviors was based on self-reported records collected from 2006 to 2010 at baseline visit. Details about the sleep behavior questions in the UK Biobank study were described elsewhere (21). Healthy sleep patterns were defined by five individual sleep behaviors (sleep duration, chronotype, insomnia, snoring, and excessive daytime sleepiness) (21). Low-risk sleep behaviors were defined as follows: sleep 7-8 h/day; early chronotype ("morning" or "morning than evening"); reported never or rarely insomnia symptoms; no self-reported snoring; and no excessive daytime sleepiness ("never/rarely" or "sometimes"). For each sleep behavior, low risk or high risk was coded as 1 or 0 , respectively. The five scores were summed to obtain a healthy sleep score ranging from 0 to 5 , with higher scores indicating a healthier sleep pattern. The participants were classified into three groups of "healthy sleep pattern" (healthy sleep scores of 4 or 5), "intermediate sleep pattern" (healthy sleep scores of 2 or 3 ), and "poor sleep pattern" (healthy sleep scores of 0 or 1 ).

\section{Definition of Genetic Risk Score}

The genotyping process and arrays used in the UK Biobank study have been reported in detail previously (23). We created the genetic risk score (GRS) for the five sleep behaviors separately using the single nucleotide polymorphisms (SNPs) reaching genome-wide association significance in the recent published genome-wide association studies (GWAS) (24-28). The information on the selected SNPs is provided in Supplementary Table 1. Individual SNP was coded as 0,1 , and 2 according to the number of risk alleles. The effect size ( $\beta$ coefficient) for each SNP was obtained from the reported GWAS data. The GRS was calculated using a weighted method $(29,30)$ : weighted $\mathrm{GRS}=\left(\beta_{1} \times \mathrm{SNP}_{1}+\beta_{2} \times \mathrm{SNP}_{2}+\ldots+\right.$ $\beta_{n} \times$ SNP $\left._{n}\right) \times(N /$ sum of the $\beta$ coefficients). We determined whether participants were at high or low genetic risk for each sleep behavior according to the median values of the corresponding GRS. Genetic risk for each sleep behavior was coded as 1 for high risk or 0 for low risk and added to calculate GRS for the overall sleep patterns. The sleep patterns GRS ranged from 0 to 5 , with higher scores indicating a higher genetic predisposition to unhealthy sleep patterns.

\section{Assessment of Outcomes}

We used the reported UK Biobank algorithms to determine T2D status (31). Hospital inpatient records were obtained through linkage to Hospital Episode Statistics for England, Scottish Morbidity Record data for Scotland, and the Patient Episode Database for Wales. Admissions and diagnoses data of the records were used to ascertain incident T2D with the ICD-10 code of E11.

\section{Statistical Analysis}

Baseline characteristics of the participants were described as means or percentages according to the quintiles of serum 250 HD levels. Follow-up time was calculated from the baseline date to diagnosis of T2D, death, or the censoring date (31 March 2017), whichever came first. Cox proportional hazards model was 
used to estimate the hazard ratio (HR) and $95 \% \mathrm{Cl}$. The proportional hazards assumptions for the Cox model were tested using Schoenfeld residuals method. Models were adjusted for age (continuous), sex (male/female), race (White European, mixed, South Asian, Black, and others), UK Biobank assessment center, average total annual household income $(<£ 18,000$, £18,000-30,999, £31,00051,999, £52,000-100,000, >£100,000, and "do not know" or missing), Townsend deprivation index (continuous), alcohol consumption (current, former, or never), smoking status (current, former, or never), BMI (kilograms per meter squared, continuous), physical activity (MET-minutes per week, continuous), healthy diet score $(0,1,2,3,4$, or 5$)$, sun exposure time in the summer (hours per day, continuous), season of blood collection (winter: December-February; spring: March-May; summer: June-August; and autumn: September-November), antihypertensive medications use (yes/no), cholesterol medications use (yes/no), and family history of diabetes (yes/no). The healthy diet score was calculated by using the following factors: vegetable intake at least four tablespoons each day (median); fruit intake at least three pieces each day (median); fish intake at least twice each week (median); unprocessed red meat intake no more than twice each week (median); and processed meat intake no more than twice each week (median). Each one point was given for each favorable diet factor, with the total diet score ranging from 0 to 5 (32). If the covariate information was missing, we imputed mean values for continuous variables or used a missingindicator approach for categorical variables such as smoking. The linear trend test was performed by treating serum $250 H D$ as a continuous variable. Stratified analyses were performed a priori by treating serum $250 \mathrm{HD}$ in quintiles according to healthy sleep patterns (healthy sleep pattern, intermediate sleep pattern, and poor sleep pattern) as well as individual sleep factors. We also conducted stratified analyses by sleep patterns GRS (low, intermediate, and high). The interaction test between serum 250HD and each category was performed by using the likelihood ratio test comparing models with and without a cross-product term.

We also conducted several sensitivity analyses to confirm the robustness of our results: additionally adjusted for milk consumption, cereal intake, and vitamin D supplement use; additionally adjusted for skin color, sunscreen use, and air pollution (particulate matter with diameters $\leq 2.5 \mu \mathrm{m}$ concentrations); and further adjusted for latitude, sleep apnea, depressive symptoms, waist circumferences, and serum glucose levels. We also conducted a subsample analysis among participants free of cardiovascular disease (CVD) and cancer at baseline or restricted case subjects with incident $T 2 D$ to $>2$ years from the baseline survey. In addition, we conducted a sensitivity analysis among participants with 25OHD deficiency ( $250 \mathrm{HD}<20 \mathrm{ng} / \mathrm{mL}$ ) (33).

All analyses were performed using SAS software (version 9.4; SAS Institute Inc., Cary, NC). All $P$ values for the tests were two-sided, and $P$ values $<0.05$ were considered as statistically significant.

\section{Data and Resource Availability}

This research was conducted using the public UK Biobank Resource.

\section{RESULTS}

Table 1 shows the baseline characteristics of the study participants according to the quintiles of serum 25OHD concentrations. Participants with higher 250HD were older, mainly of White descent, and associated with lower Townsend deprivation index. In addition, they were less likely to be current smokers, but more likely to be current drinkers and with lower BMI. They tended to have a healthy diet and more sun exposure time in the summer and were more likely to have higher physical activity levels and healthy sleep scores. Furthermore, a high prevalence of vitamin D supplementation was observed among participants with higher serum 250HD concentrations.

During a median follow-up of 8.1 years $(2,804,109$ person-years), we documented 6,940 case patients with incident T2D. We found that higher 250HD concentrations were significantly associated with a lower risk of incident T2D in the age-, sex-, and multivariate-adjusted models (Table 2). In the age- and sex-adjusted model, a $10 \mathrm{nmol} / \mathrm{L}$ increase of $250 \mathrm{HD}$ levels was associated with a $19 \%$ reduction in the risk of incident T2D $(95 \% \mathrm{Cl} 18$ $20 \%)$. After further adjustment for race, UK Biobank assessment center, household income, Townsend deprivation index, alcohol consumption, smoking status,
BMI, physical activity, healthy diet, sun exposure time in the summer, season of blood collection, antihypertensive medications use, cholesterol medications use, and family history of diabetes, 250HD levels were inversely associated with the risk of incident T2D in a doseresponse fashion. The HR $(95 \% \mathrm{Cl})$ of T2D was $0.88(0.87-0.90)$ for a $10 \mathrm{nmol} / \mathrm{L}$ increase of $250 \mathrm{HD}$; and a $49 \%$ lower risk was observed in the highest quintile compared with the lowest quintile of 250HD ( $P$ trend $<0.001$ ).

We conducted a stratified analysis according to the healthy sleep scores to evaluate whether overall sleep patterns modified the association between $250 \mathrm{HD}$ and the risk of T2D. We found a significant interaction between $25 \mathrm{OHD}$ and overall sleep patterns on the risk of incident T2D ( $P$ for interaction $=0.002)$, in which the protective HR of high 250HD was more evident among participants with higher healthy sleep scores. The HR $(95 \% \mathrm{Cl})$ of incident T2D associated with a $10 \mathrm{nmol} / \mathrm{L}$ increase of $250 \mathrm{HD}$ levels was 0.87 (0.840.89 ) among participants with a healthy sleep pattern, 0.89 (0.87-0.90) among participants with an intermediate sleep pattern, and 0.92 (0.88-0.96) among participants with a poor sleep pattern, respectively. Similar interaction patterns were observed in the analysis on the quintiles of $250 \mathrm{HD}$ (Fig. 1).

We also tested the interaction between $250 H D$ and sleep patterns GRS on the risk of incident T2D. The sleep patterns GRS was significantly correlated with healthy sleep scores $(\beta[S E]=-0.04$ [0.002]; $P<0.001)$. There was no significant interaction between sleep patterns GRS and 25OHD observed (Fig. 2).

We further assessed the interaction between 250HD and each sleep behavior separately on the risk of T2D. We found that daytime sleepiness showed a significant interaction with 250HD ( $P$ for interaction $=0.0006)($ Table 3$) . A 10 \mathrm{nmol} / \mathrm{L}$ higher 250HD level showed more prominent associations with the risk of incident T2D among participants with no frequent daytime sleepiness (HR 0.88 [95\% Cl 0.87$0.89])$ than those with excessive daytime sleepiness (HR 0.92 [95\% Cl 0.87-0.97]). In particular, the negative HRs of incident T2D for quintiles 2-5 groups compared with quintile 1 group of $250 \mathrm{HD}$ were also attenuated among participants with excessive daytime sleepiness (Fig. 3). The interactions between $25 \mathrm{OHD}$ and other 
Table 1-Baseline characteristics of participants according to quintiles of serum 25OHD levels in the UK Biobank study Quintile categories of serum 25OHD

\begin{tabular}{|c|c|c|c|c|c|c|}
\hline Characteristics & Q1 & Q2 & Q3 & Q4 & Q5 & $p^{*}$ \\
\hline $250 \mathrm{HD}(\mathrm{nmol} / \mathrm{L})$ & 22.3 & 35.9 & 47.5 & 59.7 & 80.5 & \\
\hline$N$ & 69,737 & 69,873 & 70,327 & 70,131 & 70,143 & \\
\hline Age (years) & 54.7 & 55.8 & 56.5 & 57.0 & 57.1 & $<0.001$ \\
\hline Sex, male (\%) & 45.3 & 44.9 & 45.3 & 44.7 & 44.8 & 0.03 \\
\hline Race, White (\%) & 88.2 & 94.7 & 96.9 & 98.1 & 98.8 & $<0.001$ \\
\hline BMI $\left(\mathrm{kg} / \mathrm{m}^{2}\right)$ & 28.1 & 27.7 & 27.3 & 26.8 & 26.0 & $<0.001$ \\
\hline Townsend deprivation index & -0.7 & -1.3 & -1.6 & -1.8 & -1.9 & $<0.001$ \\
\hline Household income $\geq £ 31,000(\%)$ & 46.4 & 48.9 & 48.5 & 47.7 & 47.6 & $<0.001$ \\
\hline Current drinkers (\%) & 89.1 & 92.5 & 93.6 & 94.4 & 94.8 & $<0.001$ \\
\hline Current smokers (\%) & 15.1 & 10.7 & 9.0 & 8.4 & 8.3 & $<0.001$ \\
\hline MET-minutes/week & $2,310.4$ & $2,512.3$ & $2,678.3$ & $2,837.4$ & $3,076.0$ & $<0.001$ \\
\hline Family history of diabetes (\%) & 22.9 & 21.3 & 20.1 & 19.1 & 18.0 & $<0.001$ \\
\hline Sun exposure time in summer (hours/day) & 3.4 & 3.6 & 3.8 & 3.9 & 4.2 & $<0.001$ \\
\hline Antihypertensive medications use (\%) & 19.1 & 18.6 & 18.5 & 18.5 & 18.5 & 0.01 \\
\hline Cholesterol-lowering medications use (\%) & 14.2 & 14.1 & 13.9 & 14.4 & 16.2 & $<0.001$ \\
\hline Healthy diet score (\%) & & & & & & $<0.001$ \\
\hline $0-1$ & 15.4 & 12.7 & 11.1 & 10.2 & 9.2 & \\
\hline $2-3$ & 51.6 & 49.6 & 48.5 & 47.4 & 46.4 & \\
\hline $4-5$ & 32.9 & 37.7 & 40.4 & 42.5 & 44.4 & \\
\hline Vitamin D supplementation (\%) & 2.1 & 3.5 & 5.8 & 7.7 & 10.3 & $<0.001$ \\
\hline Healthy sleep score (\%) & & & & & & $<0.001$ \\
\hline $0-1$ & 5.6 & 4.4 & 4.1 & 3.6 & 3.3 & \\
\hline $2-3$ & 59.9 & 58.5 & 57.6 & 57.0 & 56.3 & \\
\hline $4-5$ & 34.5 & 37.1 & 38.4 & 39.4 & 40.4 & \\
\hline
\end{tabular}

* $P$ values were calculated by ANOVA (continuous variables) or $\chi^{2}$ test (categorical variables).

sleep behaviors were not statistically significant.

We performed sensitivity analyses, which showed that the results were largely unchanged after further adjustment for milk consumption, cereal intake, and vitamin D supplement use or skin color, sunscreen use, particulate matter with diameters $\leq 2.5 \mu \mathrm{m}$ concentrations, sleep apnea, depressive symptoms, waist circumferences, and serum glucose levels (Supplementary Tables 24). Moreover, we observed a significant but weak association between healthy sleep patterns and north coordinate with adjustment for age, sex, and 25OHD ( $\beta$ coefficient $=-0.10$ per $1,000 \mathrm{~km}$-grid; $P<0.001)$. The association remained unchanged after restricting participants living in the same address for $>5$ years $(\beta$ coefficient $=-0.09$ per $1,000 \mathrm{~km}$-grid; $P<0.001)$. The results did not change appreciably with further adjustment for the north coordinate (Supplementary Table 4). In addition, after excluding participants with CVD or cancer at baseline or limiting participants with a follow-up time of $>2$ years, the results did not alter appreciably (Supplementary Tables 5 and 6). Besides, after further adjustment for serum $\mathrm{HbA}_{1 \mathrm{c}}$ levels or excluding participants with estimated glomerular filtration rate $<60 \mathrm{~mL} / \mathrm{min} / 1.73 \mathrm{~m}^{2}$, the results were stable. Furthermore, the results did not alter appreciably among participants with 25OHD deficiency (Supplementary Table 7). Interestingly, we observed a significant interaction between 25OHD and chronotype among participants with vitamin $D$ deficiency, as the negative HR of incident T2D associated with higher serum $250 \mathrm{HD}$ concentrations

Table 2-Adjusted HRs and 95\% CI for serum 25OHD levels with T2D in the UK Biobank study

\begin{tabular}{|c|c|c|c|c|c|c|c|}
\hline & \multicolumn{5}{|c|}{ Serum $250 H D$ (quintiles) } & \multirow{2}{*}{$\begin{array}{c}\mathrm{HR}(95 \% \mathrm{Cl}) \\
\text { for a } 10 \mathrm{nmol} / \mathrm{L} \\
\text { increase }\end{array}$} & \multirow[b]{2}{*}{$P$ trend } \\
\hline & Q1 & Q2 & Q3 & Q4 & Q5 & & \\
\hline Case subjects/ $N$ & $2,122 / 69,737$ & $1,610 / 69,873$ & $1,370 / 70,327$ & $1,028 / 70,131$ & $810 / 70,143$ & & \\
\hline Model $1^{*}$ & 1.00 & $0.70(0.66-0.75)$ & $0.57(0.53-0.61)$ & $0.41(0.38-0.44)$ & $0.32(0.30-0.35)$ & $0.81(0.80-0.82)$ & $<0.001$ \\
\hline Model $2+$ & 1.00 & $0.85(0.80-0.91)$ & $0.76(0.71-0.82)$ & $0.60(0.56-0.65)$ & $0.51(0.47-0.56)$ & $0.88(0.87-0.90)$ & $<0.001$ \\
\hline
\end{tabular}

*Adjusted for age and sex. †Adjusted for age, sex, race (White European, mixed, South Asian, Black, and others), UK Biobank assessment center, average total annual household income $(<£ 18,000, £ 18,000-30,999, £ 31,000-51,999, £ 52,000-100,000,>£ 100,000$, and "do not know" or missing), Townsend deprivation index, alcohol consumption (current, former, never, or missing), smoking status (current, former, never, or missing), BMI, physical activity (MET-minutes), healthy diet score (0,1,2,3,4, and 5), sun exposure time in the summer (hours per day), season of blood collection (winter: DecemberFebruary; spring: March-May; summer: June-August; and autumn: September-November), antihypertensive medications use (yes/no), cholesterol medications use (yes/no), and family history of diabetes (yes, no, and "do not know" or missing). 
N Case Subjects

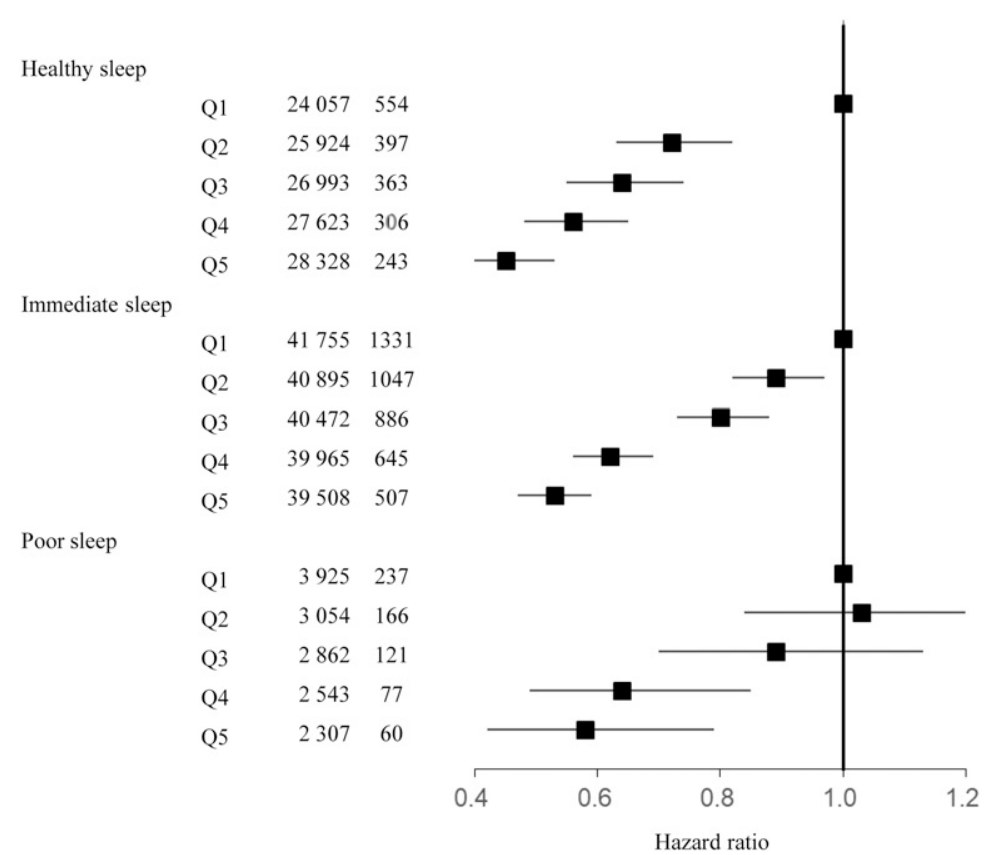

HR $(95 \%$ CI)

$\mathrm{R}(95 \% \mathrm{CI})$ for

a $10 \mathrm{nmol} / \mathrm{L}$ increase

$P$ for interaction

0.002

Figure 1-The association between serum 25OHD levels and T2D stratified by overall sleep patterns. Results were adjusted for age, sex, UK Biobank assessment center, average total annual household income $(<£ 18,000, £ 18,000-30,999, £ 31,000-51,999, £ 52,000-100,000,>£ 100,000$, and "do not know" or missing), Townsend deprivation index, alcohol consumption (current, former, never, or missing), smoking status (current, former, never, or missing), BMI, physical activity (MET-minutes), healthy diet score (0,1, 2, 3, 4, and 5), sun exposure time in the summer (hours per day), season of blood collection (winter: December-February; spring: March-May; summer: June-August; and autumn: September-November), antihypertensive medications use (yes/no), cholesterol medications use (yes/no), and family history of diabetes (yes, no, and "do not know" or missing). Q, quintile.

was attenuated among participants with late chronotype.

\section{CONCLUSIONS}

In this large-scale prospective cohort study, we observed that serum $250 \mathrm{HD}$ concentrations were inversely associated with risk of incident T2D in a doseresponse fashion, independent of traditional risk factors. We also found that the association was significantly modified by overall sleep patterns, while the decreased risk of T2D associated with high serum $250 H D$ levels appeared to be strengthened among participants with healthier sleep patterns. Among the individual sleep behaviors, daytime sleepiness showed the strongest modification effect.

Our findings of the inverse association between serum 25OHD and T2D are consistent with results from several prospective cohort studies (7-13,34). Even though the mechanisms underlying the observed inverse association between serum $250 \mathrm{HD}$ and the risk of $\mathrm{T} 2 \mathrm{D}$ remain unclear, several potential mechanisms may explain the relations. For example, vitamin D might improve $\beta$-cell function and subsequently decrease the risk of diabetes $(35,36)$. It was found that $1,25(\mathrm{OH}) 2 \mathrm{D}$, the bioactive form of $25 \mathrm{OHD}$, might upregulate the expression of insulin receptors, improve insulin responsiveness for glucose transport, and regulate calcium fluxes in the $\beta$-cell to stimulate insulin secretion (35). A recent randomized controlled trial also showed that supplementation with high-dose vita$\min D_{3}$ in patients with prediabetes and hypovitaminosis $D$ improved insulin sensitivity and reduced diabetes risk $(37,38)$. In addition, a previous study suggests that high $250 \mathrm{HD}$ concentrations may reduce $T 2 D$ risk through modulation of immune function and inflammatory processes (39). Vitamin D might regulate the proliferation, differentiation, and function of immune and inflammatory cells and, as a consequence, upregulate antiinflammatory pathways and downregulate the activation of these cells (40).

For the first time, we found that overall sleep patterns significantly modified the relations between $250 \mathrm{HD}$ and T2D risk. We recently developed an index approach to jointly evaluate overall sleep patterns (21), and we found that healthy sleep patterns, including early chronotype, sleep 7-8 h/day, never or rarely insomnia, no snoring, and no frequent excessive daytime sleepiness, were associated with a reduced risk of CVD. Our findings indicate that overall sleep patterns may better capture the relation between sleep behaviors and human health. In addition, among individual sleep behaviors, we found that daytime sleepiness showed a significant interaction with 250HD with regard to T2D risk, but not for other sleep behaviors. Notably, significant heterogeneity in the associations between 25OHD and T2D across studies was observed in previous meta-analyses. Randomized clinical trials also showed inconsistent results on whether vitamin $D$ supplementation might lower the risk of diabetes (4-6). The D2d trial yielded a nonsignificant effect of vitamin $D$ supplementation on the reduction in the incidence of T2D (4), but a recent meta-analysis of randomized clinical trials found a lower risk of T2D with vitamin D supplementation (6). Our findings suggest that varying sleep behaviors may at least partly account for such heterogeneity. 
N Case Subjects

Low genetic risk

$\begin{array}{lrrr} & \text { Q1 } & 9272 & 270 \\ & \text { Q2 } & 10242 & 200 \\ & \text { Q3 } & 10387 & 176 \\ & \text { Q4 } & 10598 & 153 \\ & \text { Q5 } & 10768 & 100 \\ & & & \\ \text { Immediate genetic } & & & \\ \text { risk } & & & \\ & \text { Q1 } & 31807 & 844 \\ & \text { Q2 } & 34116 & 747 \\ & \text { Q3 } & 35124 & 656 \\ & \text { Q4 } & 35445 & 514 \\ \text { Q5 } & 35305 & 424\end{array}$

High genetic risk

$\begin{array}{lrr}\text { Q1 } & 9476 & 273 \\ \text { Q2 } & 10115 & 222 \\ \text { Q3 } & 10481 & 184 \\ \text { Q4 } & 10483 & 171 \\ \text { Q5 } & 10737 & 117\end{array}$

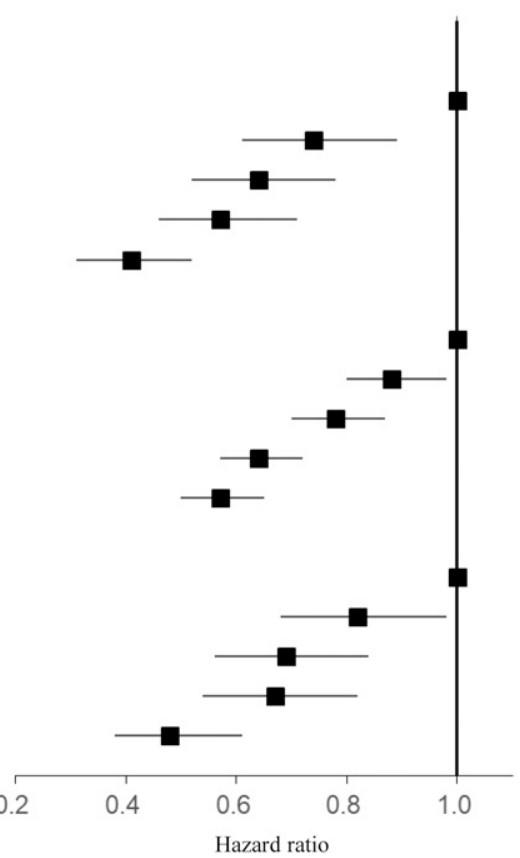

HR $(95 \%$ CI $)$<smiles>CCC</smiles>

1.00

$0.74(0.61-0.90)$

$0.64(0.52-0.78)$

$0.58(0.46-0.72)$

$0.41(0.32-0.52)$

1.00

$0.88(0.80-0.98)$

$0.78(0.71-0.88)$

$0.64(0.57-0.72)$

$0.57(0.50-0.65)$

1.00

$0.82(0.68-0.99)$

$0.69(0.56-0.84)$

$0.67(0.55-0.83)$

$0.48(0.38-0.61)$
HR $(95 \%$ CI) for

a $10 \mathrm{nmol} / \mathrm{L}$ increase

$P$ for interaction

$0.86(0.83-0.90)$

$0.90(0.88-0.91)$

$0.87(0.84-0.91)$ 
N Case Subjects
HR $(95 \%$ CI $)$

$$
\begin{gathered}
\mathrm{HR}(95 \% \mathrm{CI}) \text { for } \\
\text { a } 10 \mathrm{nmol} / \mathrm{L} \text { increase }
\end{gathered}
$$

$P$ for interaction 0.0006

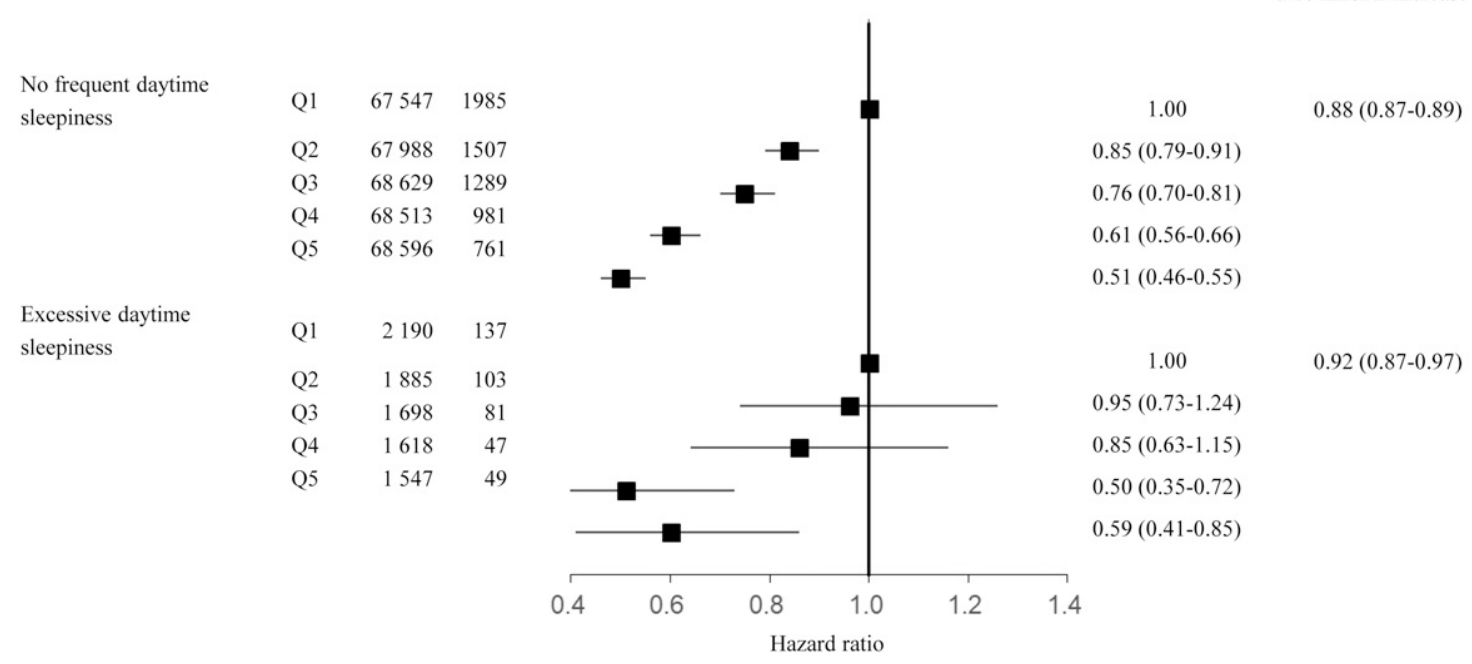

Figure 3-The association between serum 25OHD levels and T2D stratified by daytime sleepiness. Results were adjusted for age, sex, UK Biobank assessment center, average total annual household income $(<£ 18,000, £ 18,000-30,999, £ 31,000-51,999, £ 52,000-100,000,>£ 100,000$, and "do not know" or missing), Townsend deprivation index, alcohol consumption (current, former, never, or missing), smoking status (current, former, never, or missing), BMI, physical activity (MET-minutes), and healthy diet score (0,1, 2, 3, 4, and 5), sun exposure time in the summer (hours per day), season of blood collection (winter: December-February; spring: March-May; summer: June-August; and autumn: September-November), antihypertensive medications use (yes/no), cholesterol medications use (yes/no), and family history of diabetes (yes, no, and "do not know" or missing). Q, quintile.

other sleep behaviors, the negative association of high serum 25OHD with T2D appeared to be more marked among participants with a low risk of sleep duration, chronotype, insomnia, or snoring symptoms. Short sleep duration has been reported to change outdoor activities and was significantly associated with low serum $250 H D$ levels $(46,47)$. Previous studies also showed that obstructive sleep apnea, characterized by snoring symptoms, played a vital role in serum vitamin D deficiency $(16,17)$. The study also showed that subjects with more severe obstructive sleep apnea tended to present lower $250 \mathrm{HD}$ related to increased prevalence of diabetes (17).

Previous studies suggest that genetic susceptibility may modify the relations between environmental factors and human health outcomes $(48,49)$. However, we did not observe a statistically significant interaction between serum 250HD and genetically determined sleep behaviors in the study. The GRSs of the sleep patterns were calculated based on genetic variants that accounted for a small proportion of the variance in the sleep behaviors; this may partly explain the null interaction between the genetic scores and 25OHD on T2D.

\section{Strengths and Limitations}

To the best of our knowledge, this is the first prospective study to assess the interaction between 250HD and sleep behaviors on incident T2D risk. The major strengths of this study include the large sample size, the prospective design, and the well-validated measures of serum 250HD. More importantly, a wide range of covariates, including lifestyles, dietary habits, and sun exposure, was collected, which allowed for rigorous confounding adjustment. Several potential limitations, however, should be addressed. First, some case subjects with incident T2D were based on the secondary diagnosis; thus, the incident time of T2D used in the current analysis may be later than the actual onset time. Second, a single measurement of serum 250HD at baseline was used in the study, which did not take into account the changes of the biomarker during the follow-up time. However, the use of a single serum 250HD measurement in epidemiologic studies is supported by a population-based study showing that baseline $250 \mathrm{HD}$ values could predict $50 \%$ of follow-up values 14 years later ( 50$)$. Besides, a recent GWAS showed that $\sim 30 \%$ of serum $250 \mathrm{HD}$ values were heritable (51). Third, misclassification of exposures might exist since we adopted self-reported sleep data in this study. Fourth, other sleep behaviors, such as restless legs syndrome, that could interact with vitamin $D$ on the risk of T2D were not included in the healthy sleep scores. Furthermore, causality could not be determined because of the observational nature of this study, and randomized clinical trials are needed to confirm our findings. In addition, although we carefully controlled for a number of potential confounders including demographic and diet factors, lifestyles, sun exposure, as well as medical history in the analyses, residual confounding could still be present. Moreover, the current study only included participants aged 40-69 years. Since sleep behaviors and 25OHD may differ across ages, the relations among $250 H D$, sleep behaviors, and T2D in other age-groups need further studies. Finally, the current study was based on national data, and most of the participants were of European descent; thus, the generalization of the results to other populations should be interpreted with caution.

\section{Conclusion}

In summary, our study indicates that higher serum $250 \mathrm{HD}$ concentrations are associated with a lower risk of incident $\mathrm{T} 2 \mathrm{D}$, and such relations are modified by overall sleep patterns, with daytime sleepiness being the major contributor. Our findings highlight the importance to consider lifestyle factors such as sleep behaviors in investigation of the relation between vitamin $\mathrm{D}$ and human diseases. Our findings, if confirmed by replications, may have implications for the 
development of T2D prevention strategies targeting improvement of vitamin D supplementation among people with sleep disorders, especially daytime sleepiness.

Funding. This work was supported by grants from the National Heart, Lung, and Blood Institute (HL071981, HL034594, and HL126024), the National Institute of Diabetes and Digestive and Kidney Diseases (DK115679, DK091718, DK100383, and DK078616), and the Fogarty International Center (TW010790). L.Q. is a recipient of the American Heart Association Scientist Development Award (0730094N). L.Q. is also supported by National Institute of General Medical Sciences grant P20GM109036. M.W. is a recipient of a scholarship under the China Scholarship Council to pursue study in the U.S. (201906010346).

Duality of Interest. No potential conflicts of interest relevant to this article were reported. Author Contributions. M.W. and L.Q. conceived and designed the study, interpreted the data, and drafted and critically revised the manuscript. M.W. and T.Z. performed the statistical analysis. All authors contributed to the interpretation of the results and critical revision of the manuscript. All authors approved the final manuscript. L.Q. is the guarantor of this work and, as such, had full access to all of the data in the study and takes responsibility for the integrity of the data and the accuracy of the data analysis.

\section{References}

1. Bouillon R, Marcocci C, Carmeliet G, et al. Skeletal and extraskeletal actions of vitamin D: current evidence and outstanding questions. Endocr Rev 2019;40:1109-1151

2. Holick MF. Vitamin D deficiency. N Engl J Med 2007;357:266-281

3. Manson JE, Cook NR, Lee IM, et al.; VITAL Research Group. Vitamin D supplements and prevention of cancer and cardiovascular disease. N Engl J Med 2019;380:33-44

4. Pittas AG, Dawson-Hughes B, Sheehan $P$, et al.; D2d Research Group. Vitamin D supplementation and prevention of type 2 diabetes. N Engl J Med 2019;381:520-530

5. Tang H, Li D, Li Y, Zhang X, Song Y, Li X. Effects of vitamin $D$ supplementation on glucose and insulin homeostasis and incident diabetes among nondiabetic adults: a meta-analysis of randomized controlled trials. Int J Endocrinol 2018;2018: 7908764

6. Barbarawi $M$, Zayed $Y$, Barbarawi $O$, et al. Effect of vitamin D supplementation on the incidence of diabetes mellitus. J Clin Endocrinol Metab 2020;105:dgaa335

7. Buijsse $B$, Boeing $H$, Hirche $F$, et al. Plasma 25-hydroxyvitamin $D$ and its genetic determinants in relation to incident type 2 diabetes: a prospective case-cohort study. Eur J Epidemiol 2013;28:743-752

8. Lim S, Kim MJ, Choi SH, et al. Association of vitamin $D$ deficiency with incidence of type 2 diabetes in high-risk Asian subjects. Am J Clin Nutr 2013;97:524-530

9. Gagnon C, Lu ZX, Magliano DJ, et al. Serum 25-hydroxyvitamin D, calcium intake, and risk of type 2 diabetes after 5 years: results from a national, population-based prospective study (the Australian Diabetes, Obesity and Lifestyle study). Diabetes Care 2011;34:1133-1138 10. Pittas AG, Sun Q, Manson JE, DawsonHughes B, Hu FB. Plasma 25-hydroxyvitamin D concentration and risk of incident type 2 diabetes in women. Diabetes Care 2010;33:2021-2023

11. Forouhi NG, Ye Z, Rickard AP, et al. Circulating 25-hydroxyvitamin $D$ concentration and the risk of type 2 diabetes: results from the European Prospective Investigation into Cancer (EPIC)-Norfolk cohort and updated meta-analysis of prospective studies. Diabetologia 2012;55: 2173-2182

12. Song $\mathrm{Y}$, Wang $\mathrm{L}$, Pittas $\mathrm{AG}$, et al. Blood 25-hydroxy vitamin $D$ levels and incident type 2 diabetes: a meta-analysis of prospective studies. Diabetes Care 2013;36:1422-1428

13. Ekmekcioglu C, Haluza D, Kundi M. 25-Hy droxyvitamin D status and risk for colorecta cancer and type 2 diabetes mellitus: a systematic review and meta-analysis of epidemiological studies. Int J Environ Res Public Health 2017;14: 127

14. McCarty DE, Chesson AL Jr., Jain SK, Marino AA. The link between vitamin D metabolism and sleep medicine. Sleep Med Rev 2014;18:311-319 15. McCarty DE, Reddy A, Keigley Q, Kim PY, Marino AA. Vitamin D, race, and excessive daytime sleepiness. J Clin Sleep Med 2012;8:693697

16. Piovezan RD, Hirotsu C, Feres MC, et al Obstructive sleep apnea and objective short sleep duration are independently associated with the risk of serum vitamin D deficiency PLoS One 2017;12:e0180901

17. Bozkurt NC, Cakal E, Sahin M, Ozkaya EC Firat $H$, Delibasi T. The relation of serum 25hydroxyvitamin-D levels with severity of obstructive sleep apnea and glucose metabolism abnormalities. Endocrine 2012;41:518-525

18. Vu LH, Whiteman DC, van der Pols JC, Kimlin MG, Neale RE. Serum vitamin D levels in office workers in a subtropical climate. Photochem Photobiol 2011;87:714-720

19. Shan Z, Ma H, Xie M, et al. Sleep duration and risk of type 2 diabetes: a meta-analysis of prospective studies. Diabetes Care 2015;38:529-537 20. Lai YJ, Lin CL, Lin MC, et al. Population-based cohort study on the increase in the risk for type 2 diabetes mellitus development from nonapnea sleep disorders. Sleep Med 2013;14:913-918

21. Fan M, Sun D, Zhou T, et al. Sleep patterns, genetic susceptibility, and incident cardiovascular disease: a prospective study of 385292 UK Biobank participants. Eur Heart J 2020;41:11821189

22. Sudlow C, Gallacher J, Allen N, et al. UK biobank: an open access resource for identifying the causes of a wide range of complex diseases of middle and old age. PLoS Med 2015;12:e1001779 23. Bycroft C, Freeman C, Petkova D, et al. Genome-wide genetic data on $\sim 500,000$ UK Biobank participants. bioRxiv 2017;166298

24. Dashti HS, Jones SE, Wood AR, et al. Genomewide association study identifies genetic loci for self-reported habitual sleep duration supported by accelerometer-derived estimates. Nat Commun 2019;10:1100

25. $\mathrm{Hu} Y$, Shmygelska A, Tran D, Eriksson $N$, Tung JY, Hinds DA. GWAS of 89,283 individuals identifies genetic variants associated with selfreporting of being a morning person. Nat Commun 2016;7:10448

26. Lane JM, Jones SE, Dashti HS, et al.; HUNT All In Sleep. Biological and clinical insights from genetics of insomnia symptoms. Nat Genet 2019; 51:387-393

27. Jansen PR, Watanabe K, Stringer S, et al.; 23andMe Research Team. Genome-wide analysis of insomnia in 1,331,010 individuals identifies new risk loci and functional pathways. Nat Genet 2019;51:394-403

28. Wang $\mathrm{H}$, Lane JM, Jones SE, et al. Genomewide association analysis of self-reported daytime sleepiness identifies 42 loci that suggest biological subtypes. Nat Commun 2019;10: 3503

29. Ripatti S, Tikkanen E, Orho-Melander M, et al. A multilocus genetic risk score for coronary heart disease: case-control and prospective cohort analyses. Lancet 2010;376:1393-1400

30. Khera AV, Emdin CA, Drake I, et al. Genetic risk, adherence to a healthy lifestyle, and coronary disease. N Engl J Med 2016;375:2349-2358 31. Eastwood SV, Mathur R, Atkinson M, et al. Algorithms for the capture and adjudication of prevalent and incident diabetes in UK biobank. PLoS One 2016;11:e0162388

32. Pazoki R, Dehghan A, Evangelou E, et al. Genetic predisposition to high blood pressure and lifestyle factors: associations with midlife blood pressure levels and cardiovascular events [published correction appears in Circulation 2019;139:e2]. Circulation 2018;137:653-661

33. Rosen CJ, Gallagher JC. The 2011 IOM report on vitamin $\mathrm{D}$ and calcium requirements for North America: clinical implications for providers treating patients with low bone mineral density. J Clin Densitom 2011;14:79-84

34. Mitri J, Muraru MD, Pittas AG. Vitamin D and type 2 diabetes: a systematic review. Eur J Clin Nutr 2011;65:1005-1015

35. Garbossa SG, Folli F. Vitamin D, sub-inflammation and insulin resistance. $A$ window on a potential role for the interaction between bone and glucose metabolism. Rev Endocr Metab Disord 2017;18:243-258

36. Mitri J, Dawson-Hughes B, Hu FB, Pittas AG. Effects of vitamin $D$ and calcium supplementation on pancreatic $\beta$ cell function, insulin sensitivity, and glycemia in adults at high risk of diabetes: the Calcium and Vitamin D for Diabetes Mellitus (CaDDM) randomized controlled trial. Am J Clin Nutr 2011;94:486-494

37. Niroomand $M$, Fotouhi A, Irannejad N, Hosseinpanah F. Does high-dose vitamin D supplementation impact insulin resistance and risk of development of diabetes in patients with pre-diabetes? A double-blind randomized clinical trial. Diabetes Res Clin Pract 2019;148: 1-9

38. Boucher BJ. Re Niroomand M, Fotouhi A, Irannejad $\mathrm{N}$ et al. Does high-dose vitamin D supplementation impact insulin resistance and risk of development of diabetes in patients with pre-diabetes? A double-blind randomized controlled trial. Diabetes Res Clin Pract. 2019;148: 1-9 (Letter). Diabetes Res Clin Pract 2019;155: 107782

39. Cannell JJ, Grant WB, Holick MF. Vitamin D and inflammation. Dermatoendocrinol 2015;6: e983401 
40. Colotta F, Jansson B, Bonelli F. Modulation of inflammatory and immune responses by vitamin D. J Autoimmun 2017;85:78-97

41. Holick MF. Sunlight and vitamin D for bone health and prevention of autoimmune diseases, cancers, and cardiovascular disease. Am J Clin Nutr 2004;80(Suppl.):1678S-1688S

42. Daugaard S, Garde AH, Hansen AM, Vistisen HT, Rejnmark L, Kolstad HA. Indoor, outdoor, and night work and blood concentrations of vitamin $D$ and parathyroid hormone. Scand J Work Environ Health 2018;44:647-657

43. Maron FJM, Ferder L, Reiter RJ, Manucha W. Daily and seasonal mitochondrial protection: unraveling common possible mechanisms involving vitamin $D$ and melatonin. J Steroid Biochem 2020;199:105595
44. Fang $N$, Hu C, Sun W, et al. Identification of a novel melatonin-binding nuclear receptor: vitamin D receptor. J Pineal Res 2020;68: e12618

45. Ganguly G. Vitamin D deficiency, excessive daytime sleepiness: an epiphenomenon or a "chicken or an egg-which came first" issue?

Clin Sleep Med 2013;9:517-518

46. Kim JH, Chang JH, Kim DY, Kang JW. Association between self-reported sleep duration and serum vitamin $D$ level in elderly Korean adults. J Am Geriatr Soc 2014;62:2327-2332

47. Pande RU, Chandrasekhar R, Kaplish N, Rifkin DI. Low serum vitamin $D$ concentration as a predictor of short sleep duration: a NHANES 2005-2006 analysis (Abstract). Sleep 2009;32: A136
48. Qi Q, Chu AY, Kang JH, et al. Sugar-sweetened beverages and genetic risk of obesity. N Engl J Med 2012;367:1387-1396

49. Qi $Q$, Chu AY, Kang JH, et al. Fried food consumption, genetic risk, and body mass index: gene-diet interaction analysis in three US cohort studies. BMJ 2014;348:g1610

50. Jorde R, Sneve M, Hutchinson M, Emaus N, Figenschau $Y$, Grimnes $G$. Tracking of serum 25-hydroxyvitamin $D$ levels during 14 years in a population-based study and during 12 months in an intervention study. Am J Epidemiol 2010;171: 903-908

51. Revez JA, Lin T, Qiao Z, et al. Genome-wide association study identifies 143 loci associated with 25 hydroxyvitamin D concentration. Nat Commun 2020;11:1647 